\title{
Can Behcet's Disease Related Pulmonary Arterial Aneurysms be Completely Resolved?
}

\author{
Adnan Agha ${ }^{*}, 1$ Abdelhaleem M.E. Bella ${ }^{2}$, Al-Hussain M. Assiri ${ }^{1}$ and Mohammad Al-Hakami ${ }^{1}$ \\ ${ }^{I}$ Armed Forces Hospital Southern Region, Khamis Mushyt, Kingdom of Saudi Arabia \\ ${ }^{2}$ King Fahad Hospital of the University, University of Dammam, AlKhobar, Kingdom of Saudi Arabia
}

\begin{abstract}
Behçet disease is a systemic disease with diverse clinical symptoms which include, but not limited to, patients having oral and genital ulcers and eye involvement. We here report an 18-year-old male presenting with massive hemoptysis and cardiac arrest, having history of ulcers in the oral cavity and genitalia as well as having recent episode of uveitis. A pulmonary CT angiography revealed bilateral arterial aneurysms of pulmonary vessels. On receiving Immunosuppressive treatment for Behcet disease with prednisone and azathioprine over one year the pulmonary arterial aneurysms were completely resolved and the patient was discharged from the hospital albeit with persistent hypoxic brain injury from cardiac arrest.
\end{abstract}

Keywords: Behçet disease, vasculitis, pulmonary arterial aneurysm.

\section{INTRODUCTION}

Behçet disease (BD) is a multisystem disorder characterized by vasculitis, first described in 1937, classically consisting of recurrent ulceration of oral and genital mucosa with relapsing uveitis [1]. However since its inception, involvement of other organs like skin, central nervous system, gastrointestinal, and pulmonary have been reported; including subcutaneous thrombophlebitis, deep vein thrombosis, epididymitis, arterial occlusion and/or aneurysms, arthralgia, arthritis, as well as renal complications [2]. The pulmonary complications of BD have classified into three groups (1) pulmonary artery aneurysm (PAA), (2) pulmonary parenchymal changes, and (3) a miscellaneous group consisting including pulmonary artery occlusion, pleural effusion, and pulmonary obstructive airway disease etc. [3]. Pulmonary artery vasculitis itself is rare; affects mainly young men presenting with dyspnoea, cough, chest pain and haemoptysis. It carries a bad prognosis in patients with $\mathrm{BD}$; although systemic glucocorticoid and cyclophosphamide pulse therapy have shown some limited benefit in some case reports [4]. In a recent study of 534 patients with Behçet disease, only 8 suffered from pulmonary aneurysms but 6 of these died despite immunosuppressive treatment or surgery, underlining the mortality associated with PAA [5]. A study by Hamuryudan et al. presents the data of 26 patients having $\mathrm{BD}$ with PAA, showing an improved survival rate of $62 \%$; secondary to prompt diagnosis of PAA and early commencement of long term immunosuppression with cyclophosphamide and steroids [6]. The patients with PAA presenting with profuse haemoptysis had the worst prognosis in the study [6].

*Address correspondence to this author at the Department of Internal Medicine, Armed Forces Hospital Southern Region, PO Box 101 Khamis Mushyt, Kingdom of Saudi Arabia; Tel: +966566880913;

E-mail: adnanagha@hotmail.com
We attempt here to describe a case of an 18-year-old male with $\mathrm{BD}$ and PAA seen on CT pulmonary angiography resolved with steroid and azathioprine treatment over one year.

\section{CASE REPORT}

An 18 year old Saudi unmarried male student had been attending the ophthalmology clinic for posterior uveitis for the last few weeks. He was also followed in urology clinic for testicular pain with benign hypo-echoic lesions, presumed to be trauma related, for the last few months. He presented to the emergency room with massive bleeding from mouth, (query hemoptysis versus hematemesis) and found to have cardiac arrest on arrival to the emergency room. The patient had no history of any allergies, surgeries in the past, sexual contacts, smoking, addiction or any familial illnesses. Cardiopulmonary resuscitation was done and he was intubated, ventilated and shifted to intensive care unit. His laboratory data on arrival revealed no significant abnormalities. His endotracheal tube and nasogastric tube continued to drain over $500 \mathrm{ml}$ of bloody aspirate over the next 24 hours, putting the possibility of asphyxia from aspiration of blood as a likely cause of initial cardiac arrest.

$\mathrm{He}$ underwent esophagogastrodudenoscopy which showed coffee ground material in stomach but no signs of active bleeding. His toxicology screening and autoimmune profile (rheumatoid factor, antinuclear antibodies) and viral $\&$ Brucella serology were all negative. The patient remained intubated and mechanically ventilated with a baseline Glasgow coma scale of 7 . His CT (computerized tomography) scan chest with contrast was negative for pulmonary embolism. His echocardiogram on the $2^{\text {nd }}$ post admission was initially suspicious for probable mass versus vegetation over tricuspid valve which prompted IV antibiotics (vancomycin and amikacin) as well as antifungal (amphotericin B) as patient remained culture negative throughout his stay. However, the second echocardiography 
and cardiac surgery review refuted the need for any surgical intervention for suspected tricuspid lesion due to poor clinical condition of the patient and the paucity of chances for successful repair. The patient remained afebrile from the $3^{\text {rd }}$ post admission day onwards, which prompted the primary physician to discontinue all antibiotics/antifungal after 14 days as all septic screening remained negative throughout. His initial CT brain revealed hypoxic brain injury with minimal cerebral edema which resolved on subsequent scans. He also remained under the auspices of neurologist for two episodes of myoclonic seizures treated with phenytoin and maintained on sodium valproate. An opinion regarding possible biopsy of the hypo-echoic lesion in the testicles was sought but the urologist's impression remained that the lesions are likely secondary to a traumatic cause which require no further workup.

As the diagnosis remained elusive, the family was again called for obtaining additional points in history. On $14^{\text {th }}$ post admission day, during re-interview with the family about recent oral ulcer that the patient had now developed in the mouth while in intensive care unit, they revealed that the patient has recurrent oral and genital ulcers on almost a monthly basis along with recurrent joint pains off and on. His Pathergy test was done which was inconclusive. His ophthalmological examination was significant for posterior uveitis. Rheumatologist's opinion was sought and it was mutually agreed by the multidisciplinary team to treat the patient with steroids as a case of Behcet's disease. A second CT chest with contrast was done with reconstruction imaging, which showed massive pulmonary arteriovenous malformations/aneurysm (see Fig. 1) and left small pulmonary infarct without any evidence of thromboembolism. His steroids were changed to Methylprednisolone 1 gram intravenous pulse therapy for 5 days in addition to initiation of azathioprine $50 \mathrm{mg}$. The family was counseled but did not agree to consent for cyclophosphamide therapy due to potential side effects. The steroids were then tapered down over 6 months and maintained on $10 \mathrm{mg}$ prednisolone with azathiprine $75 \mathrm{mg}$ daily along with osteoporosis prevention medications.

The patient was eventually extubated and tracheostomy closed but continued to have irreversible brain hypoxic injury with minimal responsiveness with eye contact and nodding and no motor response in the limbs. He remained under follow-up with cardiology, rheumatology, nephrology and neurology for over one year as family was unwilling to take the patient home. His biochemistry remained normal throughout the stay. His repeat ultrasound revealed that the testicular lesions had completely resolved while the repeat echocardiography revealed that the tricuspid lesion had also completely resolved, all indicative of possible vasculitic origin. His repeat CT scan chest showed all pulmonary arterial aneurysms resolved after almost one year of steroids and azathioprine (see Fig. 2). The patient was discharged with home health care and over the last one year follow-up he has remained in an unchanged condition, bedridden with persistent hypoxic brain injury with no new lesions or other active problems.

\section{DISCUSSION}

This case is an example of how the vasculitic lesions of Behcet's disease (BD) can present and their likely response with immunosuppressants. This is a rare presentation of Behcet's disease with pulmonary arterial aneurysm presenting as massive hemoptysis causing cardiac arrest. Our patient had presented with multiple pulmonary arterial aneurysms secondary to vasculitis fulfilling the BD criteria. There was no evidence of any connective tissue disorder.

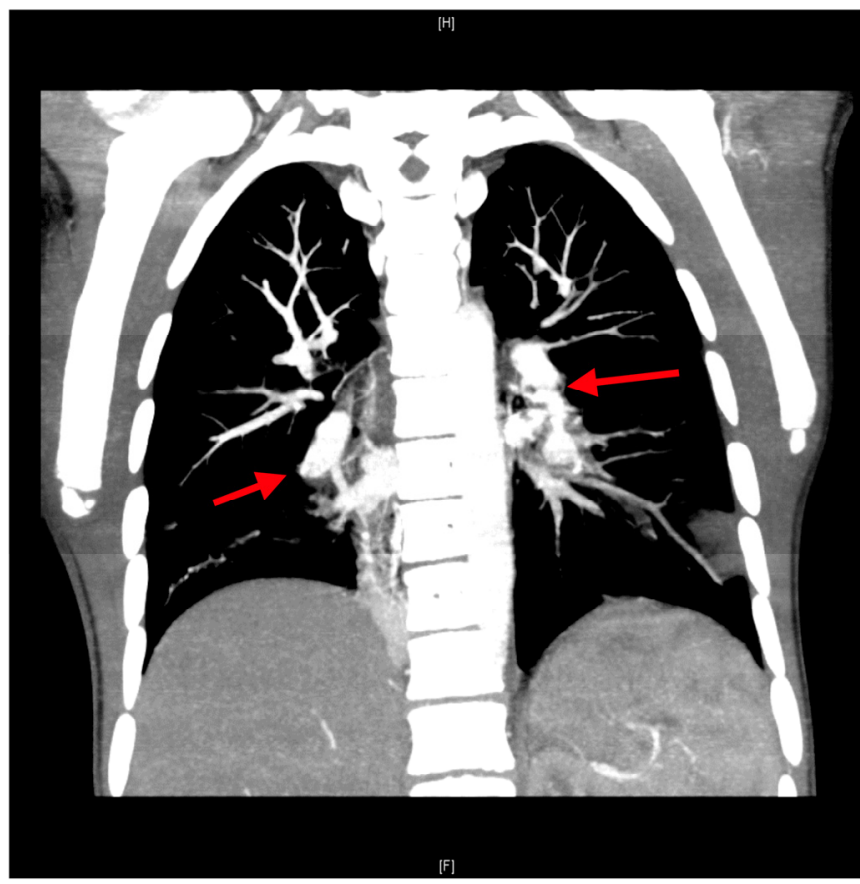

Fig. (1). CT angiography of the chest showing multiple pulmonary arterial aneurysms with left pulmonary infarction.

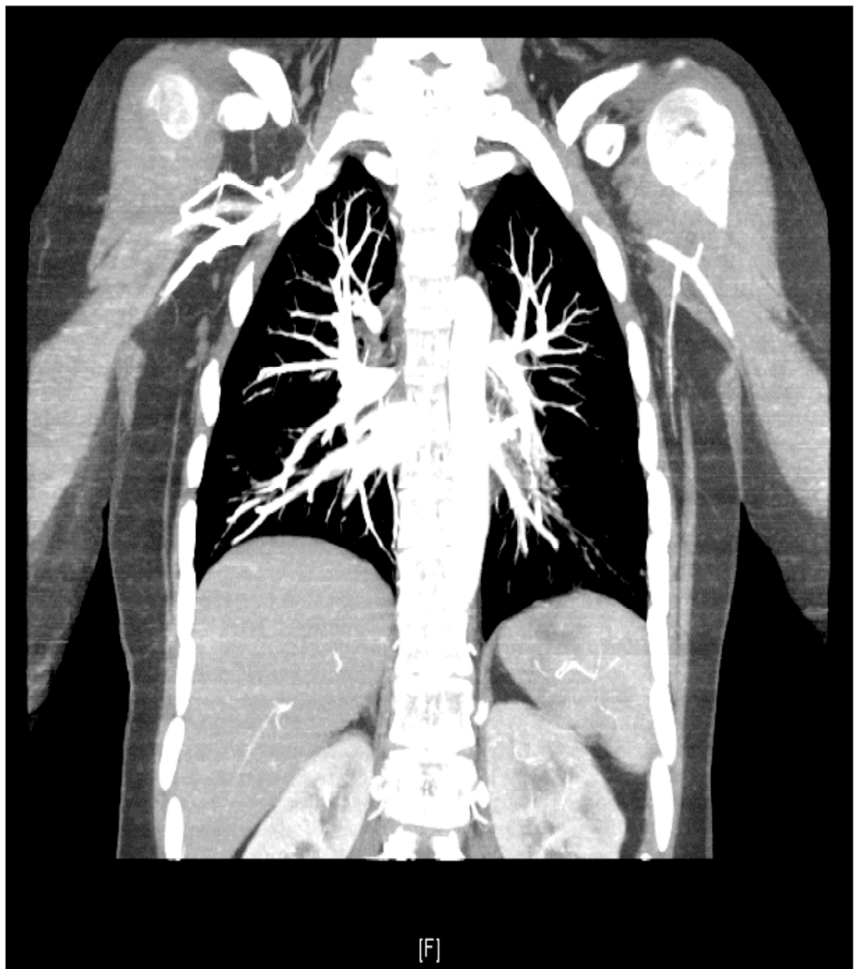

Fig. (2). CT angiography of the chest showing no pulmonary artery aneurysm.

Pulmonary arterial aneurysm (PAA) is the most common type of pulmonary involvement in BD [7]. Since vascular 
involvement in $\mathrm{BD}$ is more frequent among male patients, male gender is a risk factor for pulmonary arterial aneurysm [8]. Hemoptysis is the most common symptom of PAA [9]. PAA may be single or multiple, unilateral or bilateral, true or false and can be complicated by bronchopulmonary artery fistulas [10]. Studies performed in Turkey $(\mathrm{n}=2,147)$ [11], Iran $(\mathrm{n}=3,153)[12]$, Japan $(\mathrm{n}=3,316)[13]$, and Europe $(\mathrm{n}$ $=714)$ [14], have shown that the frequencies of vascular involvement (including pulmonary arterial aneurysms) in BD were $17 \%, 9 \%, 9 \%$, and 10 to $37 \%$, respectively.

Pulmonary arterial aneurysm of one the causes of deaths in BD [15]. Hamuryudan et al. reported that 12 of 24 patients $(50 \%)$ died after an average of 10 months after the onset of hemoptysis [16]. The overall mortality of BD in a study of 817 patients was found to be $5 \%$ with male sex, $\mathrm{PAA}$, and the number of BD flares being strongly associated with mortality [17]. Another large cohort study over 20 years shows mortality in $\mathrm{BD}$ patients being over $9.8 \%$ with male sex, central nervous system involvement and PAA being poor prognostic factors [18]. A variety of other treatment modalities, such as surgery, anticoagulation, embolization, and more effectively of them all immunosuppression especially with cyclophosphamide and high dose steroids have been used in the management of pulmonary arterial aneurysm with limited success [19-21]. In one such case report, a 39-year-old man with $\mathrm{BD}$ who presented with hemoptysis with computed tomography and magnetic resonance angiography revealing multiple, bilateral pulmonary arterial aneurysms; a course of cyclophosphamide and corticosteroid therapy resulted in complete resolution of his radiologic findings [4].

So can Behcet's disease related pulmonary arterial aneurysms be completely resolved? The answer to this question is probably yes but we probably need more large scale randomized trials of steroid and immunosuppressant therapy for BD with pulmonary arterial aneurysms for more valid results and generalized inference.

\section{ACKNOWLEDGEMENT}

None declared.

\section{CONFLICT OF INTEREST}

None declared.

\section{REFERENCES}

[1] Behçet H. Uber rezidivierende aphthose, durch ein virus verursachte Geschwure am Mund, am Auge und an den Genitalien. Dermatol Woschenschr 1937; 105: 1152-7.
[2] Shimizu T, Ehrlich GE, Inaba G, Hayashi K. Behçet's disease (Behçet's syndrome). Semin Arthritis Rheum 1979; 8: 223-60.

[3] Yurdakul S, Hamuryudan V, Yazici H. Behcet's syndrome. Curr Opin Rheumatol 2004; 16: 38-42.

[4] Aktogu S, Erer OF, Urpek G, Soy O, Tibet G. Multiple pulmonary arterial aneurysms in Behcet's disease: clinical and radiologic remission after cyclophosphamide and corticosteroid therapy. Respiration 2002; 69: 178-81.

[5] International Study Group for Behcet's Disease. Criteria for diagnosis of Behçet's disease. Lancet 1990. 335; 1078-80.

[6] Hamuryudan $\mathrm{V}, \mathrm{Oz} \mathrm{B}$, Tüzün $\mathrm{H}$, Yazici $\mathrm{H}$. The menacing pulmonary artery aneurysms of Behçet's syndrome. Clin Exp Rheumatol 2004; 22: S1-3.

[7] Le Thi Huong D, Wechsler B, Papo T, et al. Arterial lesions in Behcet's disease: a study in 25 patients. J Rheumatol 1995; 22: 2103-13.

[8] Ahn JM, Im JG, Ryoo JW, et al. Thoracic manifestations of Behcet syndrome: radiographic and $\mathrm{CT}$ findings in nine patients. Radiology 1995; 194: 199-203.

[9] Numan F, Islak C, Berkmen T, Tüzün H, Cokyüksel O. Behcet disease: pulmonary arterial involvement in 15 cases. Radiology 1994; 192: 465-8.

[10] Köse AA, Kayabalı M, Sarıca R, Saglik E, Azizlerli G. Pulmonary artery involvement in Behçet's disease. In: Zouboulis CC, Ed. Proceedings of the 10th International Conference on Behcet's disease. New York, NY: Kluwer Academic/Plenum Publishers 2003; pp. 419-22.

[11] Yilmaz S, Cimen KA. Pulmonary artery aneurysms in Behçet's disease. Rheumatol Int 2010; 30: 1401-3.

[12] Davatchi F, Shahram F, Chams-Davatchi C, et al. Behcet's disease in Iran: analysis of 6500 cases. Int J Rheum Dis 2010; 13: 367-73.

[13] Takahama M, Yamamoto R, Nakajima R, Tada H. Successful surgical treatment of pulmonary artery aneurysm in Behçet's syndrome. Interact Cardiovasc Thorac Surg 2009; 8: 390-2.

[14] Salvarani C, Pipitone N, Catanoso MG, et al. Epidemiology and clinical course of Behçet's disease in the Reggio Emilia area of Northern Italy: a seventeen-year population-based study. Arthritis Rheum 2007; 57: 171-8.

[15] Yazici H, Başaran G, Hamuryudan V, et al. The ten-year mortality in Behçet's syndrome. Br J Rheumatol 1996; 35: 139-41.

[16] Hamuryudan V, Er T, Seyahi E, et al. Pulmonary artery aneurysms in Behçet syndrome. Am J Med 2004; 117: 867-70.

[17] Saadoun D, Wechsler B, Desseaux K, et al. Mortality in Behçet's disease. Arthritis Rheum 2010; 62: 2806-12.

[18] Kural-Seyahi E, Fresko I, Seyahi N, et al. The long-term mortality and morbidity of Behçet syndrome: a 2-decade outcome survey of 387 patients followed at a dedicated center. Medicine (Baltimore). 2003; 82: 60-76.

[19] Calamia KT, Schirmer M, Melikoglu M. Major vessel involvement in Behçet's disease: an update. Curr Opin Rheumatol 2011; 23 : 2431.

[20] El Houari T, Oukerraj L, Ghzaiel L, et al. Management of Behçet disease with multiple complications. Hellenic J Cardiol 2009; 50: $420-2$.

[21] Uzun O, Akpolat T, Erkan L. Pulmonary vasculitis in behcet disease: a cumulative analysis. Chest 2005; 127: 2243-53. 